\title{
Covid-19 Pandemia, Impact on Food Industry and Nutrition
}

ISSN: 2640-9208

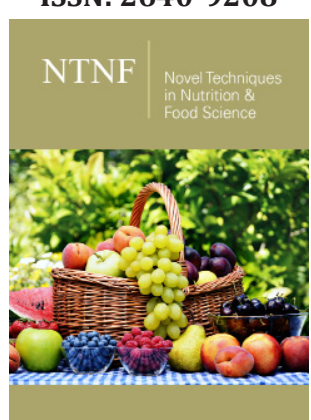

*Corresponding author: Kıvılcım Çelik, Department of Food Engineering, Turkey.

Submission: 洞 October 23, 2020

Published: 洯 November 24, 2020

Volume 5 - Issue 3

How to cite this article: Kıvılcım Çelik, Aslıhan Tüğen, Bülent Ergönül. Covid-19 Pandemia, Impact on Food Industry and Nutrition. Nov Tech Nutri Food Sci. 5(3). NTNF. 000612. 2020

DOI: 10.31031/NTNF.2020.05.000612

Copyright@ Kıvılcım Çelik. This article is distributed under the terms of the Creative Commons Attribution 4.0 International License, which permits unrestricted use and redistribution provided that the original author and source are credited.

\author{
Kıvılcım Celik*, Aslıhan Tüğen and Bülent Ergönül \\ Department of Food Engineering, Turkey
}

\begin{abstract}
Since the first month of 2020, the whole world has faced an epidemic not seen since the Spanish flu pandemic. The new type of coronavirus, COVID-19. It is an easily transmissible disease caused by SARSCoV-2, first identified in December 2019 and declared as a pandemic by the World Health Organization on March 11, 2020. As of October 2020, 37 million cases and more than 1 million deaths from COVID-19 have been recorded worldwide. The COVID-19 pandemic has had a very significant and negative impact on every area from global trade to individual life and economy due to the measures taken against the epidemic and the social restrictions that must be implemented. The food sector is also the most important among the groups affected by this pandemic. In this COVID-19 pandemic, as in every disease, nutrition has a very important place for human health.
\end{abstract}

Keywords: COVID-19;Pandemic;Nutrition;Food sector

\section{Introduction}

The whole world has faced an epidemic since the first month of 2020 that has not been seen since the Spanish flu pandemic. The new type of coronavirus, COVID-19. It is an easily transmissible disease caused by SARS-CoV-2, first identified in December 2019 and declared as a pandemic by the World Health Organization on March 11, 2020. Since the first infection case was linked to the Huanan Seafood Market (Wuhan, China), it was initially featured in the media as the Wuhan virus [1]. Zhou et al. [2] It has been revealed that the virus is transmitted to humans by consumption of bats, SARS-CoV-2 and bat coronavirus have a gene sequence similarity of up to $96.2 \%$, while it has been determined that the starting point of the infection is due to the consumption of bat and pangolin and that it is passed on to humans through consumption of these creatures. As of October 2020, 37 million cases and more than 1 million deaths from COVID-19 have been recorded worldwide. It is known that COVID-19 infections are seen in more than 200 countries where these numbers are increasing exponentially every day. Flu-like symptoms of COVID-19 usually appear 5-6 days after infection and manifest themselves with symptoms such as cough, sore throat, fever, muscle and body aches, and loss of smell and / or taste [1,3].

COVID-19 poses a major threat to countries suffering from poverty and poor healthcare infrastructure. It continues to threaten the masses in South America, especially in India, some African countries and Brazil, due to the weak health infrastructure. Tedros Adhanom Ghebreyesus, Director General of the World Health Organization, explains that their biggest concerns are the potential of the virus to spread to countries with weaker health systems, and points out that there is a significant risk, especially for the refugee population today. Today, more people than ever since the Second World War have chosen to become refugees and leave their countries in order to escape from violence, conflict and persecution. Most of those who have left their country live in camps, the refugee population living close to each other and lacking adequate medical facilities are at a particularly high risk from COVID-19. It is thought that the disease may pose five main threats in terms of food security [4]. The first of these is that the epidemic has become a bigger threat for some countries due to poverty and insufficient health infrastructure, followed by the possibility of more severe consequences in countries without a strong social security system. Another important threat is that those with chronic or acute nutritional deficiencies are more likely to be affected by this epidemic. 
The probability of spreading extreme poverty and hunger due to breakdowns in food supply chains, food shortages and sudden increases in prices and the slowdown or stagnation of the global economy are also perceived as important threats.

\section{Covid-19 Pandemic, Its Effects on the Food Industry and the Importance of Nutrition}

COVID-19 is fatal for people suffering from chronic or acute hunger or malnutrition due to weak immunity. It is widely known that especially the elderly or those with chronic illnesses are those who suffer the most from COVID-19 infection. In addition to these, it is necessary to consider the weakened immune system caused by inadequate and unbalanced nutrition as an important factor. During and after the Ebola epidemic, many scientific sources state that there is a linear relationship between patient survival and good nutrition and strong immune systems. As a result of the restrictions announced by the increase in the number of cases and deaths worldwide, especially in European countries, in midMarch 2020, there have been interruptions in food supply chains, difficulties in accessing certain foods due to stocking and significant and sudden increases in food prices. Due to the consumers' desire to make their breads at home, their access to basic food and food additives such as flour and yeast has been restricted and the prices of these products have increased significantly. At the beginning of the restrictions and curfews, the same situation was observed in pasta consumption. People have resorted to resolving their hesitations about accessing food by stocking up, and consumers have become difficult to access certain foods in some regions due to excessive food intake and storage. A similar situation occurred during the Ebola epidemic in 2014, leading to dramatic increases in prices of basic food in affected countries in West Africa [5]. Moreover, increases in food prices, export restrictions, speculation and panic behavior in the market were partly responsible for the dramatic and sudden increase in global food prices at that time. Millions of families in many developing countries normally spend more than half of their income on food. Countries that rely heavily on imported food to meet demand, including sub-Saharan Africa, have been adversely affected by supply chain disruptions, especially in the face of the closure of border crossings. Finally, the economies of developing countries were also adversely affected as agricultural activities were negatively affected by interruptions and disruptions in non-food supply chains such as fertilizers and other critical inputs. In many countries where the economy slowed or contracted, hunger increased mostly in middle-income countries, while economic decline, poverty and inadequate practices in the field of food security accompanied each other and continued their negative effects.

It is thought that the disease may pose five main threats in terms of food security [4]. The first of these is that the epidemic has become a bigger threat for some countries due to poverty and insufficient health infrastructure, followed by the possibility of more severe consequences in countries without a strong social security system. Another important threat is that those with chronic or acute nutritional deficiencies are more likely to be affected by this epidemic. The probability of spreading extreme poverty and hunger due to breakdowns in food supply chains, food shortages and sudden increases in prices and the slowdown or stagnation of the global economy are also perceived as important threats.

In the early days of the coronavirus pandemic, consumers experienced problems in accessing food in most countries. The food supply chain in our country gave a very successful test in the face of this global epidemic, and there were no problems in consumers' access to food except for the first few days of restrictions. Countries that have implemented physical distance regulations have also taken measures to protect access to food and have not so far experienced major cuts in food supplies. Although there were difficulties in accessing some foodstuffs in the first days of curfews in our country, these negativities disappeared in a short time due to the active production and services of food logistics companies. At this point, perhaps one of the most important facts to be addressed is waste. Approximately 1.5 billion tons of food is wasted every year in the world [6]. In our country, approximately 20 million tons of food is wasted annually and thrown away [7]. It is a known fact that the most wasteful consumption of fruits and vegetables in our country, which ranks 14th in Europe in terms of sustainable agriculture. When households are considered, fruit and vegetables are the most wasteful losses, followed by cereals, oilseeds, and legumes. It is thought that, especially during epidemic periods, the household economy will be less affected, and the reduction of household food waste will have an important effect for efficient access to food.

Another household habit observed especially in this process is that consumers tend to focus on foods with a longer shelf life and more virtual media are used for food shopping than in the past, and a tendency to shop from e-commerce sites has emerged. As consumers prefer to cook and eat at home in this process, it is thought that there will be a significant change in food demand, preference and supply. Accordingly, it is inevitable that food prices will increase. A balanced diet is very important during and after an infection. Infections damage the body, especially as they cause fever, and therefore the body needs extra energy and nutrients. Maintaining a healthy diet is essential during the COVID-19 pandemic. While no food or dietary supplement can prevent COVID-19 infection, it should be noted that maintaining a healthy diet is an important part of supporting a strong immune system. Sufficient and balanced nutrition, physical exercise, regular and adequate sleep and stress coping strengthen the immune system. As of October 2020, there is no vaccine or specific drug developed against COVID-19 yet. Considering that the process of finding the vaccine and its spread afterwards will be quite long, it is important for consumers to review their eating habits in order to keep their immune systems resistant.

It is of great importance to gain a balanced diet rich in vegetables and fruits in order to regularly supply copper, folic acid, iron, selenium, zinc and vitamins A, B6, B12, C and D, which play an 
important role in a strong immune system [8]. In addition, drinking enough water, exercising, walking as much as possible, and getting enough sleep by providing sleep patterns are the most important factors in maintaining and maintaining immunity. Drinking plenty of water (6-8 glasses a day for most adults) also helps our immune system. Drinking only water instead of sugar-sweetened beverages also helps reduce the risk of consuming too many calories to maintain a healthy weight [8]. Fresh fruits and vegetables play an important role as a source of sufficient fiber, minerals and vitamins for a healthy diet. Frozen fruits and vegetables serve the same purpose if they are properly stored and thawed. Grains, especially whole grains, nuts and legumes as well as fats rich in unsaturated fatty acids are indispensable elements of a balanced diet. A diet supported by such foods plays a very important role in protecting the immune system. In addition to a balanced diet, consumers should be re-aware of the pandemic process in order to avoid some habits that damage the immune system such as alcohol and smoking.

Although COVID-19 cases are seen less among children, today approximately 1.5 billion children have suspended their education as a result of the measures and restrictions taken due to the pandemic. The break in education around the world has restricted not only children's right to education but also adequate and balanced food in schools. Adequate nutrition is essential for the health and well-being of schoolchildren. Organizations such as the World Health Organization (WHO), FAO and UNICEF continue to work to take a series of measures regarding the nutrition of school-age children and the safety of school food after the break in education is over and the return to normal begins.

\section{Conclusion}

The global COVID-19 outbreak has brought with it many socioeconomic problems and dilemmas. Among them, there is definitely a food safety weakness. Especially in the first months of the epidemic and following restrictions, there was a difficulty in "accessing food", which was also significantly affected by the consumers' tendency to stockpile, and this situation reduced its impact with the measures taken. However, this epidemic process has shown that securing food production and supply should be an inevitable priority. Encouraging good agricultural practices throughout the world and especially in our country, and even making them a legal obligation, ensuring that every consumer has access to food at a healthy and affordable price, based on the concepts of food security and sovereignty, especially in such epidemics, in order to ensure the resistance of the immune system by providing adequate and balanced nutrition of the consumer. It should be one of the top priorities. In this respect, the COVID 19 pandemic has been an important stimulus to take the aforementioned steps.

\section{References}

1. Galanakis CM (2020) The food systems in the Era of the Coronavirus (COVID-19) Pandemic Crisis. Foods 9(4): 523.

2. Zhou P, Yang XL, Wang XG, Hu B, Zhang L, et al. (2020) A pneumonia outbreak associated with a new coronavirus of probable bat origin. Nature 579(7798): 270-273.

3. Ahn DG, Shin HJ, Kim MH, Lee S, Kim HS, et al. (2020) Current status of epidemiology, diagnosis, therapeutics, and vaccines for novel coronavirus disease 2019 (COVID-19). J Microbiol Biotechnol 30(3): 313-324.

4. https://insight.wfp.org/covid-19-and-the-5-major-threats-it-poses-toglobal-food-security-1c4da2ff6657

5. Sova C (2020) Coronavirus and the 5 major threats it poses to global food security virus risks undermining eorts to reverse trend of rising hunger. World Food Programme, USA.

6. https://www.bcg.com/publications/2018/tackling-1.6-billion-tonfood-loss-and-waste-crisis

7. https://www.trthaber.com/haber/yasam/bakan-pakdemirli-her-yil188-milyon-ton-gida-cope-gidiyor-486236.html

8. Anon (2020) Maintaining a healthy diet during the COVID-19 pandemic. Food and Agriculture Organization of the United Nations, pp. 1-3. 